University of Nebraska - Lincoln

DigitalCommons@University of Nebraska - Lincoln

Barry Chin Li Cheung Publications

Published Research - Department of Chemistry

4-27-2001

\title{
Energy Gaps in "Metallic" Single-Walled Carbon Nanotubes
}

Min Ouyang

Harvard University, Cambridge, MA

Jin-Lin Huang

Harvard University, Cambridge, MA

Chin Li Cheung

University of Nebraska at Lincoln, ccheung2@unl.edu

Charles Lieber

Harvard University, Cambridge, MA

Follow this and additional works at: https://digitalcommons.unl.edu/chemistrycheung

Part of the Chemistry Commons

Ouyang, Min; Huang, Jin-Lin; Cheung, Chin Li; and Lieber, Charles, "Energy Gaps in "Metallic" Single-Walled Carbon Nanotubes" (2001). Barry Chin Li Cheung Publications. 20.

https://digitalcommons.unl.edu/chemistrycheung/20

This Article is brought to you for free and open access by the Published Research - Department of Chemistry at DigitalCommons@University of Nebraska - Lincoln. It has been accepted for inclusion in Barry Chin Li Cheung Publications by an authorized administrator of DigitalCommons@University of Nebraska - Lincoln. 
Published in Science Vol. 292. no. 5517 (April 27, 2001), pp. 702-705; doi: 10.1126/science.1058853

Copyright (C) 2001 by the American Association for the Advancement of Science. Used by permission.

http://www.sciencemag.org/cgi/content/full/292/5517/702

Submitted January 8, 2001; accepted March 6, 2001.

REPORTS

\title{
Energy Gaps in "Metallic" Single-Walled Carbon Nanotubes
}

\author{
Min Ouyang, ${ }^{1}$ Jin-Lin Huang, ${ }^{1}$ Chin Li Cheung, ${ }^{1}$ and Charles M. Lieber ${ }^{1,2,}$ \\ 1 Department of Chemistry and Chemical Biology, Harvard University, Cambridge, MA 02138, USA \\ 2 Division of Engineering and Applied Sciences, Harvard University, Cambridge, MA 02138, USA \\ * Corresponding author; email: $\underline{\mathrm{cml} @ \text { cmliris.harvard.edu }}$
}

\begin{abstract}
Metallic single-walled carbon nanotubes have been proposed to be good one-dimensional conductors. However, the finite curvature of the graphene sheet that forms the nanotubes and the broken symmetry due to the local environment may modify their electronic properties. We used low-temperature atomically resolved scanning tunneling microscopy to investigate zigzag and armchair nanotubes, both thought to be metallic. "Metallic" zigzag nanotubes were found to have energy gaps with magnitudes that depend inversely on the square of the tube radius, whereas isolated armchair tubes do not have energy gaps. Additionally, armchair nanotubes packed in bundles have pseudogaps, which exhibit an inverse dependence on tube radius. These observed energy gaps suggest that most "metallic" single-walled nanotubes are not true metals, and they have implications for our understanding of the electronic properties and potential applications of carbon nanotubes.
\end{abstract}

Are "metallic" single-walled carbon nanotubes (SWNTs) true metals (1)? This question has attracted theoretical interest (2-13) and is of increasing practical importance because of the potential applications of SWNTs in nanoelectronic devices $(14,15)$. Tight-binding calculations carried out for the two-dimensional (2D) graphene sheet model predict that SWNTs with indices $(n, n$ $+3 q$ ) (where $q$ is an integer) should behave as metallic quantum wires with high electrical conductivity $(2-4,15)$. However, the finite curvature of SWNTs alters the overlap of $\pi$-electron wave functions, and this could produce small energy gaps in "metal- lic" zigzag $(n, 0)$ (where $n$ is a multiple of 3 ) and chiral SWNTs at the Fermi energy $\left(E_{\mathrm{F}}\right)$ $(3-8)$. In addition, intertube interactions can break the rotational symmetry of armchair SWNTs $(n, n)$, which allows mixing of $\pi$ and $\pi^{*}$ bands, and has been predicted to lead to a pseudogap at $E_{\mathrm{F}}(9-13)$.

Direct experimental data addressing these predictions have been lacking, although the nature of nanotube electronic density of states (DOS) near $E_{\mathrm{F}}$ is clearly critical to our understanding of electrical transport through these materials. We have used low-temperature scanning tunneling microscopy (STM) to character- ize the atomic structure and local DOS of metallic zigzag and armchair SWNTs. The STM measurements were carried out in ultrahigh vacuum at $\sim 5 \mathrm{~K}$ on SWNT samples supported on $\mathrm{Au}(111)$ substrates as described (16-18). Particular care was taken to minimize solution processing of the SWNTs (which can deposit impurities and/or dope the tubes) and to remove absorbed gases in the ultrahigh-vacuum environment before sample cooling. Atomic resolution was routinely achieved on the SWNTs, and more than 20 zigzag and armchair SWNTs were characterized in detail in this study. 
Analysis of a typical atomically resolved STM image of a SWNT in a bundle (Figure 1A) shows that the chiral angle $\theta$ [where $\theta$ is defined relative to the $(n, 0)$ zigzag direction, i.e., $\theta=0$ for zigzag SWNTs] is zero and the diameter $d$ is $1.17 \pm 0.06 \mathrm{~nm}$. This angle defines the SWNT as an achiral zigzag tube, and from the diameter constraint we assign indices of $(15,0)$. In general, the high quality and stability of the image data have enabled straightforward assignment of the indices of the SWNTs investigated in this study. Consistency in the assignment of indices was checked by comparing the measured tunneling conductance data, which were acquired by tunneling spectroscopy simultaneously with the images, with the DOS calculated using a $\pi$-only tight-binding calculation for the assigned indices $(17,18)$. The agreement between the measured and calculated van Hove singularity peaks is excellent (Figure 1B).

The spectroscopy data (Figure 1B) also show previously unobserved features near $E_{\mathrm{F}}$ in $(n, 0)$ SWNTs where $n / 3$ is an integer-that is, "metallic" zigzag tubes. Specifically, the data recorded on $(9,0),(12,0)$, and $(15,0)$ tubes show gap-like structures at $E_{\mathrm{F}}$. High-resolution current-voltage (I-V) and conductance-voltage $[(d I / d V)-V]$ spectra were acquired to elucidate the nature of these gap-like structures. Spectra measured from more than 10 distinct locations in each tube and from distinct tubes with the same indices were found to be very reproducible. In representative plots of $I-V$ and $(d I / d V) /(I /$ $V)$ (Figure 1C), which is proportional to the local DOS, a complete suppression of the DOS near $E_{\mathrm{F}}$ with sharp increases at energies that depend on the zigzag tube radius can be seen. The widths of these gaps were extracted (19) from the measured spectra to yield averaged energies of $0.080 \pm 0.005,0$ $.042 \pm 0.004$, and $0.029 \pm 0.004 \mathrm{eV}$ for $(9$, $0),(12,0)$, and $(15,0)$ SWNTs, respectively. Therefore, these "metallic" zigzag tubes are in fact small-gap semiconductors.

The inverse dependence of the gap magnitude on tube radius implies that these gaps arise from curvature in the graphene sheet (20). To gain more physical insight into these small gaps, we have developed a model that treats the curvature-induced shift of the Fermi wave vector $\left(\mathbf{k}_{\mathrm{F}}\right)$ using an approach similar to that for deformed SWNTs (21). Briefly, finite curvature reduces the overlap of nearest-neighbor $\pi$ orbitals by a factor of $\cos ^{2}(\alpha)$ (Figure 2B), which shifts $\mathbf{k}_{\mathrm{F}}$ from the first Brillouin zone corner (Kpoint) of a $2 \mathrm{D}$ graphene sheet. For "metallic" zigzag tubes, $\mathbf{k}_{\mathrm{F}}$ moves away from the $\mathrm{K}$-point along the circumferential direction c (Figure 2B) such that the allowed onedimensional subband $\mathbf{k}$ no longer passes through $\mathbf{k}_{\mathrm{F}}$; that is, a small gap opens at $E_{\mathrm{F}}$. The model further predicts that the small energy gaps of "metallic" zigzag tubes should scale as $A_{\mathrm{o}} / R^{2}$ with $A_{\mathrm{o}}=3 \gamma_{0} a_{\mathrm{cc}}{ }^{2} / 16$, where $R$ is the tube radius, $\gamma_{0}$ is the magnitude of the tight-binding transfer matrix element, and $a_{\mathrm{cc}}=0.142 \mathrm{~nm}$ is the carbon-carbon bond distance. This result is consistent with previous full-valence tight-binding calculations (6) and analytical calculations for a Hamiltonian on a curved surface (8).

Our experimental data fit well the predicted $A_{\mathrm{o}} / R^{2}$ dependence (Figure 2A) and yield a $\gamma_{0}$ value of $2.60 \mathrm{eV}$. This value of $\gamma_{0}$ is in good agreement with the range of 2.5 to $2.7 \mathrm{eV}$ determined in previous STM studies $(16-18,22)$ and with values determined
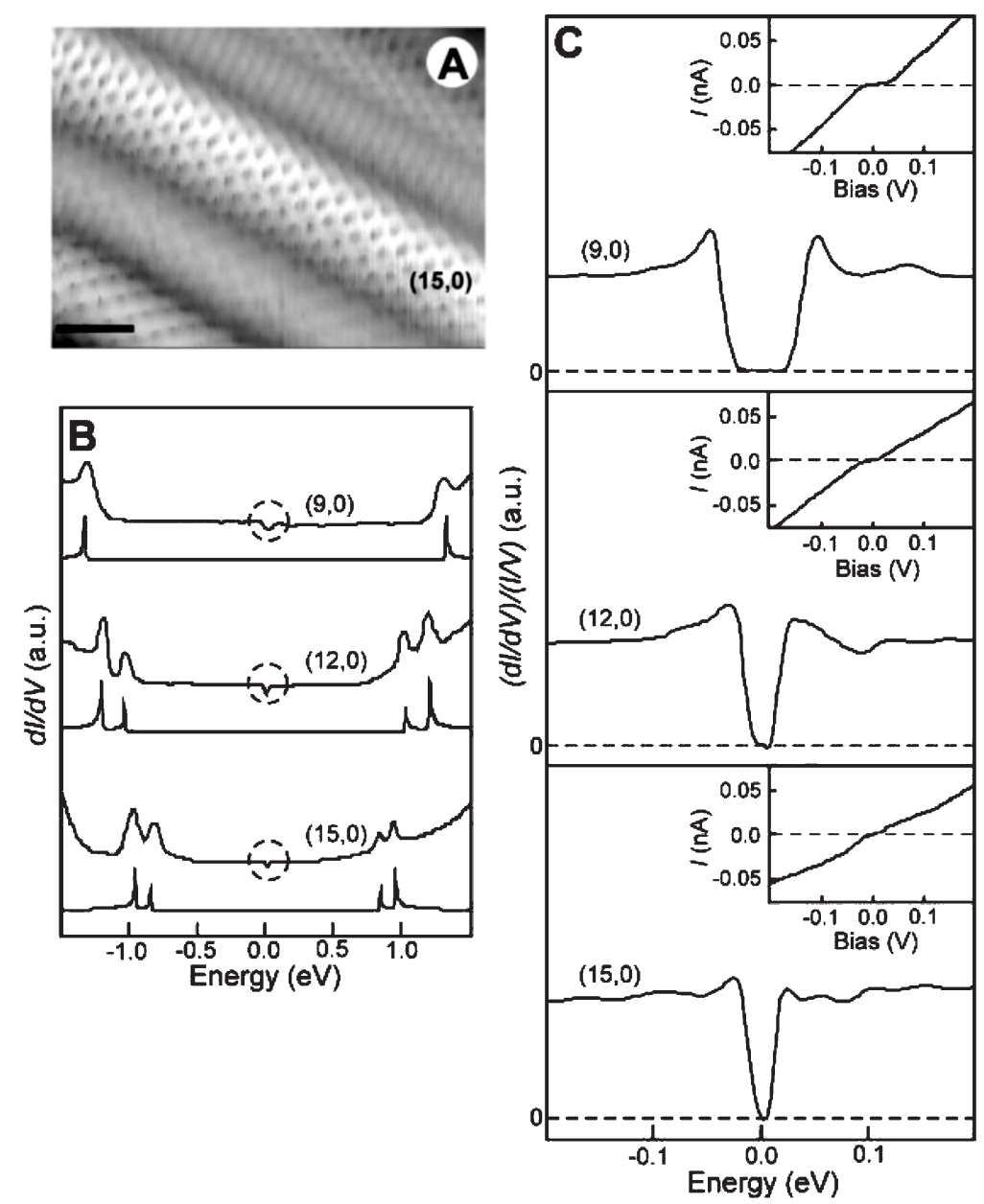

Figure 1. Atomic structure and spectroscopy of "metallic" zigzag SWNTs. (A) Typical atomically resolved STM image of a $(15,0)$ SWNT. The image was recorded in the constant-current mode with bias voltage of $0.65 \mathrm{~V}$ and current $I=0.15 \mathrm{nA}$. Scale bar, $1 \mathrm{~nm}$. (B) Tunneling conductance data, $d I / d V$, for different zigzag SWNTs, with corresponding calculated DOS shown below each experimental curve (a.u., arbitrary units). The data were recorded as the in-phase component of $I$ directly by a lock-in amplifier with a $7.37-\mathrm{kHz}$ modulation signal of $2 \mathrm{mV}$ peak-to-peak amplitude to the bias voltage. The new features in the low-energy region of the $(9,0),(12,0)$, and $(15,0)$ tubes are highlighted by dashed circles. (C) Typical high-resolution normalized conductance $(\mathrm{dl} / \mathrm{dV}) /(\mathrm{I} / \mathrm{V})$ curves and measured $I-\mathrm{V}$ curves (insets) for $(9,0),(12,0)$, and $(15,0)$ tubes, respectively. The $(d l / d V) /(I / V)$ curves were calculated from $d I / d V$ and $I-V$ data.

from first-principles calculations (7), and thus provides an additional consistency

In previous STM studies, it was suggested that the absence of small-gap structures could be due to temperature or vestigations show that the SWNT-substrate interaction does not obscure these intrinsic small-gap features and that the magnitudes of the gaps in "metallic" zigzag SWNTs are sufficiently large that they should have been observable. A more likely reason for the failure to observe these gaps previously is
that the aforementioned STM measurements were made on predominantly chiral metallic tubes with $|\theta|>0$. Because the predicted 
gaps decrease rapidly as $|\theta|$ increases from zero $(6,8)$, thermal excitations and instrumental noise could readily obscure intrinsic small-gap features in the chiral tubes. This $\theta$ dependence underscores the importance of making atomically resolved measurements in order to resolve and understand the role of curvature.

We have also carried out measurements on a number of $(n, n)$ armchair SWNTs, which are expected to be true metals because of the crossing of $\pi$ and $\pi^{*}$ bands at $E_{\mathrm{F}}$ $(2-4,15,18)$. Atomically resolved images of an $(8,8)$ SWNT in a bundle and an $(8,8)$ tube isolated on a $\mathrm{Au}(111)$ substrate surface (Figure 3 , A \& B) are representative of those analyzed. Comparison of the tunneling spectra with the calculated DOS of an isolated $(8,8)$ SWNT (Figure 3C) shows good agreement for the positions of the van Hove singularity peaks for the $(8,8)$ tubes both in the bundle and isolated on the substrate. These data suggest that isolated and bundle tubes have very similar electronic band structures for energies greater than $0.1 \mathrm{eV}$ (the positions of the van Hove singularities are essentially the same), and thus suggest that tube-tube interactions do not perturb the electronic band structure features at larger energy scales.

Tunneling spectra recorded on the $(8,8)$ SWNT in the bundle also exhibit a gap-like feature at $E_{\mathrm{F}}$ that is not present in spectra of the isolated $(8,8)$ armchair tube. High-resolution measurements (Figure 3D) show unambiguously that there is a reproducible gap in the DOS at $E_{\mathrm{F}}$ in the bundle armchair SWNT, whereas there is no such feature for the structurally identical tube isolated on the substrate (i.e., the DOS is finite and constant around $\left.E_{\mathrm{F}}\right)$. The gap feature observed in the $(8,8)$ tube is substantial $(\sim 100 \mathrm{meV})$ but distinct from the curvature-induced gaps discussed above, because the DOS are suppressed but not reduced completely to zero at $E_{\mathrm{F}}$. For this reason, we term this feature a pseudogap. We have observed similar pseudogap structures in a number of other armchair SWNTs in bundles (six independent tubes characterized in total) (Figure 4A). Overall, the magnitudes of the gaps range from $\sim 80$ to $100 \mathrm{meV}$ in the $(10,10)$ through $(7,7)$ SWNTs characterized in our studies, and these magnitudes exhibit an inverse dependence on tube radius.

Our results for armchair SWNTs are consistent with recent theoretical studies (913) but also show differences. Qualitatively, the origin of the pseudogap can be understood by considering the $\pi$ and $\pi^{*}$ bands. In an isolated armchair SWNT, the $\pi$ and $\pi^{*}$ bands have different parity under a reflection operation and cross at $E_{\mathrm{F}}$ without opening a

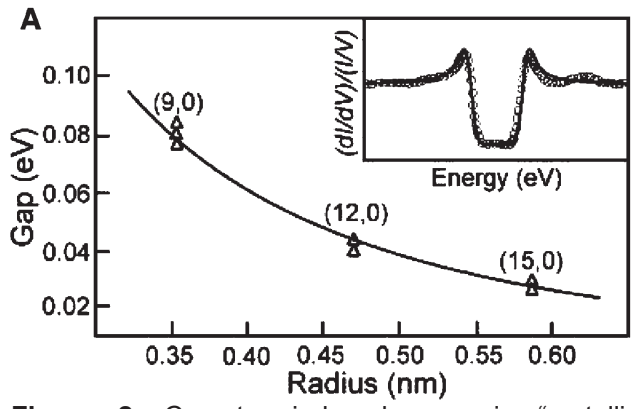

B

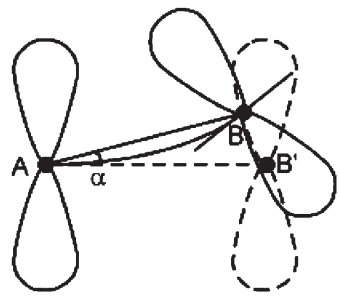

Figure 2. Curvature-induced gaps in "metallic" zigzag SWNTs. (A) Energy gaps versus tube radius. Every data point (triangle) represents the averaged gap value for one distinct $(n, 0)$ tube; the solid line corresponds to a fit of $3 \mathrm{Y}_{0} \mathrm{a}_{\mathrm{cc}}{ }^{2}$ $\left(16 R^{2}\right)$ with $Y_{0}=2.60 \mathrm{eV}$. Inset shows a typical fit of the DOS (solid line) to the experimental data (circles) for a $(9,0)$ tube with $\delta=0.006 \mathrm{eV}(19)$. (B) Schematic of the shift of $\mathbf{k}_{\mathrm{F}}$ for a curved graphene sheet. Upper panel: A and B' represent the parallel $\pi$ orbitals of two nearest-neighbor carbon atoms on a flat graphene sheet, B denotes the $\pi$ orbital of a nearestneighbor atom on a curved surface, and $\alpha$ is defined as the angle between $A B$ and $A B^{\prime}$ and represents a measure of curvature. Lower panel: The bold lines represent the two sides of the first Brillouin zone of a flat graphene sheet, the thin parallel lines are the allowed one-dimensional $k$-states, and $\mathbf{c}$ is the circumferential direction perpendicular to the tube axis. In "metallic" zigzag SWNTs, $\mathbf{k}_{\mathrm{F}}$ moves along the $\mathbf{c}$ direction away from the K-point.
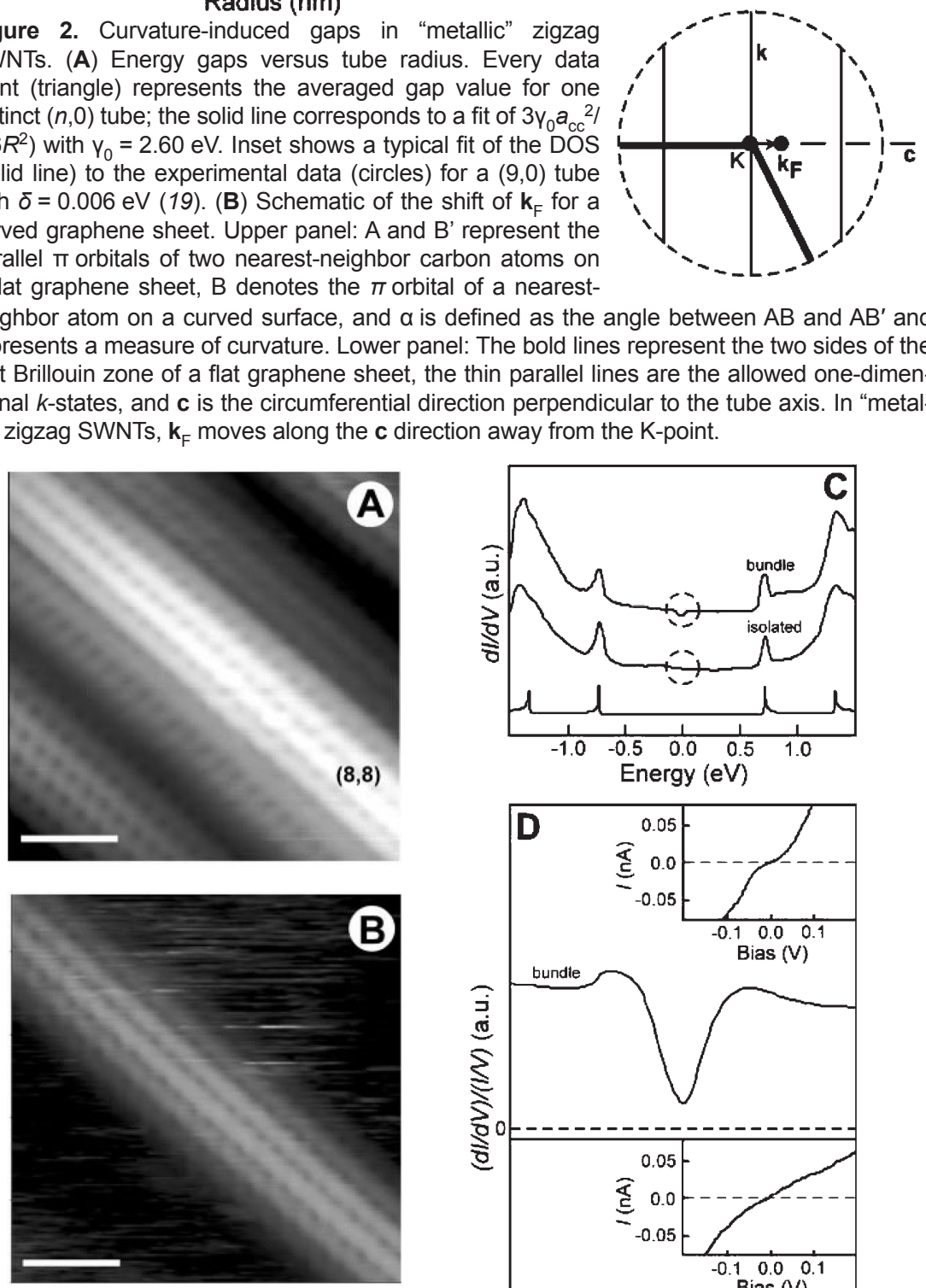

Figure 3. Atomic structure and spectroscopy of armchair SWNTs. (A) STM image of an $(8,8)$ armchair SWNT exposed on the top of a bundle with index marked, recorded
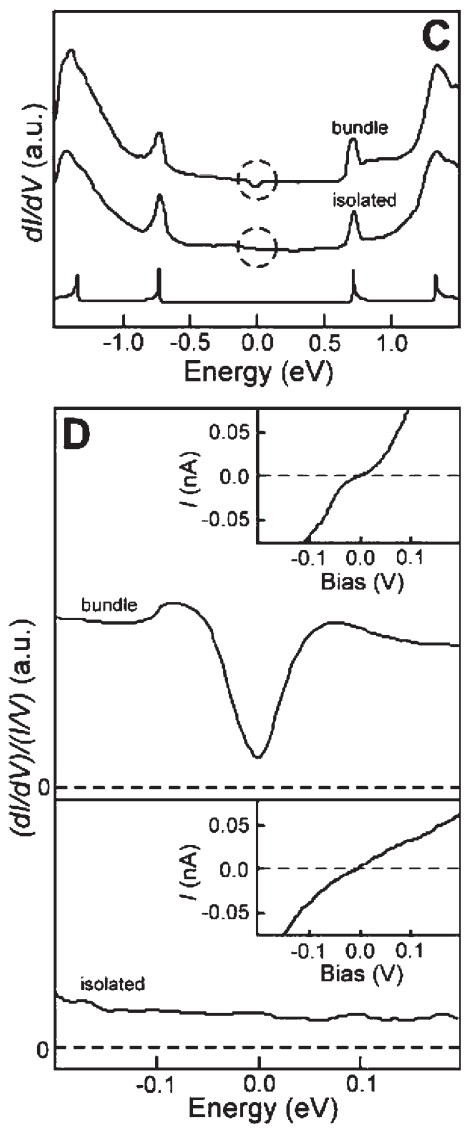

in constant-current mode with bias voltage of $0.60 \mathrm{~V}$ and tunneling current of $0.15 \mathrm{nA}$. (B) STM image of isolated $(8,8)$ SWNTs on $\mathrm{Au}(111)$ substrate, recorded in constant-current mode with bias voltage of $0.55 \mathrm{~V}$ and tunneling current of $0.10 \mathrm{nA}$. Scale bars, $1 \mathrm{~nm}$. (C) $d I / d V$ recorded on the $(8,8)$ armchair SWNTs in $(A)$ and $(B)$. The calculated DOS for an isolated $(8,8)$ tube is displayed below the experimental data. The dashed circles highlight the relevant low-energy region for both $(8,8)$ tubes. (D) Typical high-resolution $(d I / d V) /(I / V)$ and measured $I-V$ (inset) data in the low-energy region. 
gap (7) (Figure 4B, upper panel). However, when the $n$-fold rotational symmetry is broken by, for example, strong tube-tube interactions (9-12), the $\pi^{*}$ and $\pi$ bands can mix, which produces a pseudogap at $E_{\mathrm{F}}$ (Figure 4B, lower panel). Comparison of our experimental results with calculations carried out by four independent groups (9-13) shows good agreement for $(8,8)$ indices $(12)$ and some deviations for $(10,10)$ indices $(9-11$, 13). This suggests that the basic physics underlying pseudogap formation is correct, although a complete understanding will require more accurate simulation of the experimental conditions. For example, the $(n, n)$ tubes exhibiting pseudogaps in our studies are on the surface of a bundle, whereas the theoretical studies considered tubes in crystalline or completely disordered bundles. In addition, it is important to recognize that our results show that interactions between isolated armchair SWNTs and Au(111) substrates (Figure 3B) are sufficiently weak that they do not perturb the SWNT band structure near $E_{\mathrm{F}}$.

Our experimental results also show that the magnitude of the pseudogap depends inversely on SWNT radius (Figure 4A). A recent theoretical study of $(n, n)$ SWNTs in a trigonal lattice (13) suggests that the magnitude of the pseudogap depends inversely on the square root of the tube radius (dashed line, Figure 4A). This prediction is in qualitative agreement with our results, although the quantitative

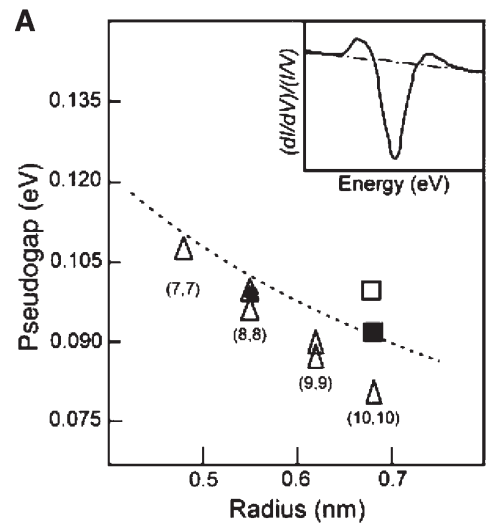

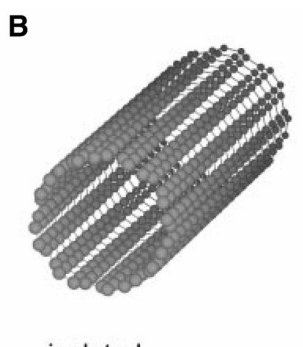

isolated

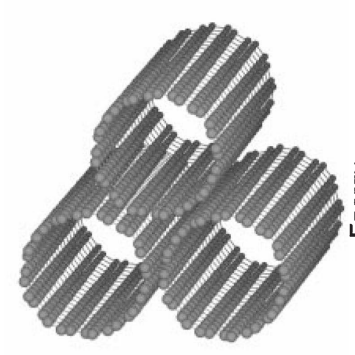

bundle agreement is poor; that is, our data exhibit a stronger size dependence. It is possible that the greater $\pi$-orbital extension outside tubes with smaller radii (5) leads to stronger tube-tube interactions (and hence larger pseudogaps) than in tubes with larger radii, although additional work will be necessary to better understand the underlying physics.

The presence of sizable gaps in "metallic" zigzag and armchair SWNTs shows that the electronic properties of these materials differ substantially from the expectations of simple models, and that this difference should be considered in the interpretation of electrical and optical measurements and in applications of SWNTs in nanoelectronics. The complete gaps in $(n, 0)$ zigzag tubes show that these materials are semiconductors. Additionally, the large pseudogaps in armchair tubes in bundles will modify electrical transport, and the very low DOS at $E_{\mathrm{F}}$ will make extended states in such tubes susceptible to localization. From a positive standpoint, the presence of the pseudogap in armchair SWNTs should make these samples sensitive to doping, which may enable the exploitation of this phenomenon in sensor applications. Our results also show that isolated armchair SWNTs have neither gaps or pseudogaps; thus, by controlling the local environment of armchair tubes, it will be possible to maximize their conductivity - for example, as interconnects in molecular electronics.

Figure 4. Pseudogaps in armchair SWNTs. (A) Summary of the observed energy gaps versus tube radius. Each experimental data point (triangle) represents an average gap value measured on a distinct $(n, n)$ tube. In the inset, the dot-dash line is a fit to the background of the normalized conductance data; the gap value is defined by the intersection of this dashed line and the experimental DOS curve. Theoretical results are also shown for comparison: The solid square and dashed line correspond, respectively, to the gap value of a $(10,10)$ tube and radius dependence of the gap widths $(13)$; the open square is the result for a $(10,10)$ tube from $(9)$; and the solid circle shows the value calculated for an $(8,8)$ tube $(12)$. The $(10,10)$ result from (11), $200 \mathrm{meV}$, is off the scale. (B) Upper panel: Model of an isolated $(8,8)$ tube and corresponding schematic of the crossing $\pi$ and $\pi^{*}$ bands at $E_{\mathrm{F}}$. Lower panel: Model of a bundle of three $(8,8)$ tubes and corresponding band diagram near $E_{F}(9,12)$.

\section{REFERENCES \& NOTES}

1. A SWNT can be viewed as a cylinder formed by rolling up a strip cut from an infinite graphene sheet. The geometric structure of SWNTs is represented by an index $(n, m)$, which corresponds to the roll-up vector $\mathbf{C}_{\mathrm{h}}=n \mathbf{a}_{1}+m \mathbf{a}_{2}$, where $\mathbf{a}_{1}$ and $\mathbf{a}_{2}$ are the unit vectors of the graphene sheet [M. S. Dresselhaus, G. Dresselhaus, P. C. Ekund, Science of Fullerenes and Carbon Nanotubes (Academic Press, San Diego, CA, 1996)].

2. J. W. Mintmire, B. I. Dunlap, C. T. White, Phys. Rev. Lett. 68, 631 (1992).

3. N. Hamada, S. Sawada, A. Oshiyama, Phys. Rev. Lett. 68, 1579 (1992).

4. R. Saito, M. Fujita, G. Dresselhaus, M. Dresselhaus, Phys. Rev. B 46, 1804 (1992).

5. X. Blasé, L. X. Benedict, E. L. Shirley, S. G. Louie, Phys. Rev. Lett. 72, 1878 (1994).

6. C. T. White, D. H. Robertson, J. W. Mintmire, in Clusters and Nanostructured Materials, P. Jena, S. Behera, Eds. (Nova, New York, 1996), pp. 231-237.

7. J. W. Mintmire, D. H. Robertson, C. T. White, J. Phys. Chem. Solids 54, 1835 (1993).

8. C. L. Kane and E. J. Mele, Phys. Rev. Lett. 78, 1932 (1997)

9. P. Delaney, H. J. Choi, J. Ihm, S. G. Louie, M. L. Cohen, Nature 391, 466 (1998).

10. , Phys. Rev. B 60, 7899 (1999).

11. Y.-K. Kwon, S. Saito, D. Tomanek, Phys. Rev. B 58, 13314 (1998).

12. A. Rubio, Appl. Phys. A 68, 275 (1999).

13. A. A. Maarouf, C. L. Kane, E. J. Mele, Phys. Rev. B 61, 11156 (2000).

14. P. L. McEuen, Nature 393, 15 (1998).

15. C. Dekker, Phys. Today 52(5), 22 (1999).

16. T. W. Odom, J.-L. Huang, P. Kim, C. M. Lieber, Nature 391, 62 (1998)

17. P. Kim, T. W. Odom, J.-L. Huang, C. M. Lieber, Phys. Rev. Lett. 82, 1225 (1999).

18. T. W. Odom, J.-L. Huang, P. Kim, C. M. Lieber, J. Phys. Chem. B 104, 2794 (2000).

19. The gap values were obtained by fitting the experimental $(d I / d V) /(I / V)$ data with a universal function of $D_{0} \cdot|E| /\left(E^{2}-\Delta^{2}\right)^{1 / 2}$ broadened by a Gaussian convolution to account for instrumental effects and temperature: $\rho(E)=D_{0}$ ' $\left[\delta(2 \pi)^{1 / 2}\right] \cdot \int\left[|\varepsilon| /\left(\varepsilon^{2}-\Delta^{2}\right)^{1 / 2}\right] \cdot\left\{\exp \left[-(\varepsilon-E)^{2} /\right.\right.$ $\left.\left.2 \delta^{2}\right]\right\} d \varepsilon$, where $D_{0}$ is a constant for a specific tube, $2 \Delta$ is the gap width, $\delta$ is the broadening parameter, and $\varepsilon$ is the integral variable with energy units. The universal function was derived by the approach of J. W. Mintmire and C. T. White [Phys. Rev. Lett. 81, 2506 (1998)] (M. Ouyang, J.-L. Huang, C. M. Lieber, unpublished data). The inset of Figure 2A shows such a typical fit.

20. The effects of tube bending, local defects, and radial tube deformation can be excluded because (i) all of the SWNTs characterized were straight, and local defects were not observed in the atomically resolved images; and (ii) the tube diameters were sufficiently small that deformation can be ruled out [ J. Tersoff and R. S. Ruoff, Phys. Rev. Lett. 73, 676 (1994); T. Hertel, R. E. Walkup, Ph. Avouris, Phys. Rev. B 58, 13870 (1998)].

21. L. Yang and J. Han, Phys. Rev. Lett. 85, 154 (2000).

22. J. W. G. Wildoer, L. C. Venema, A. G. Rinzler, R. E. Smalley, C. Dekker, Nature 391, 59 (1998).

23. We thank H. Park, J. Wang, L. J. Lauhon, M. S. Gudiksen, T. W. Odom, and P. Kim for helpful discussions. Supported by NSF grant DMR-9813399. 